\title{
High Speed Data Processing for Imaging MS-Based Molecular Histology Using Graphical Processing Units
}

\author{
Emrys A. Jones, ${ }^{1}$ René J. M. van Zeijl, ${ }^{1}$ Per E. Andrén, ${ }^{2}$ André M. Deelder, ${ }^{1}$ \\ Lex Wolters, ${ }^{3}$ Liam A. McDonnell ${ }^{1}$
}

${ }^{1}$ Biomolecular Mass Spectrometry Unit, Department of Parasitology, Leiden University Medical Center, Albinusdreef 2, 2333ZA, Leiden, The Netherlands

${ }^{2}$ Department of Pharmaceutical Biosciences, Medical Mass Spectrometry, Uppsala University, Uppsala, Sweden

${ }^{3}$ Leiden Institute of Advanced Computer Science, Leiden University, Leiden, The Netherlands

\begin{abstract}
Imaging MS enables the distributions of hundreds of biomolecular ions to be determined directly from tissue samples. The application of multivariate methods, to identify pixels possessing correlated MS profiles, is referred to as molecular histology as tissues can be annotated on the basis of the MS profiles. The application of imaging MS-based molecular histology to larger tissue series, for clinical applications, requires significantly increased computational capacity in order to efficiently analyze the very large, highly dimensional datasets. Such datasets are highly suited to processing using graphical processor units, a very cost-effective solution for high speed processing. Here we demonstrate up to $13 \times$ speed improvements for imaging MS-based molecular histology using off-the-shelf components, and demonstrate equivalence with CPU based calculations. It is then discussed how imaging MS investigations may be designed to fully exploit the high speed of graphical processor units.
\end{abstract}

Key words: Imaging mass spectrometry, Molecular histology, Graphical processor units, GPU, Bioinformatics

\section{Introduction}

$\mathrm{M}$ atrix assisted laser desorption/ionization mass spectrometry (MALDI-MS) can generate MS profiles directly from tissue that contain hundreds of distinct biomolecular ions [1]. Spatially-correlated analysis, imaging MS, can simultaneously reveal how the intensity of each of these biomolecular ions varies across the tissue sample [2]. There is growing evidence that imaging MS is having an impact in disease detection, particularly cancer [3]. The differential MS profiles found in tumors can be used to identify candidate biomarkers [4], and when combined with clinical outcomes identify MS signatures associated with prognosis [5].

One of the advantages of imaging MS is that it can annotate tissues based on their MS profiles and thereby distinguish

Correspondence to: Liam A. McDonnell; e-mail: 1.a.mcdonnell@lumc.nl biomolecularly different regions even if they are not distinct using established histologic and histochemical tools. Imaging MS-based molecular histology has been used to differentiate between histologically overlapping/identical tumors [6], found evidence of intratumor heterogeneity that could indicate clonal development [6-8] and revealed altered protein expression at tumor interface zones [9, 10]. These capabilities offer enormous potential to investigate biomolecular changes that occur prior to (or without) morphological change, or those for which a molecular specific stain is unavailable.

Imaging MS experiments are recorded as a list of discrete spectra, each associated with a specific pixel. MS images are created by displaying the intensities of an MS peak, from each spectrum, at their pixel locations as a false color image. When subjected to multivariate or cluster analysis, to identify regions with distinct MS profiles, the imaging MS data is analyzed as the list of discrete spectra. The imageoutputs of the multivariate/cluster analysis are obtained by 
projecting each spectrum's score onto its pixel location as a false color image.

If multiple tissue sections, mounted on to the same MALDI target plate, are analyzed within a single data acquisition sequence the imaging MS data is still a list of pixel-associated spectra but the pixel coordinates reflect the different locations of the tissues. We have adapted this approach to enable the datasets of multiple tissue sections, recorded on different days (and on different MALDI target plates), to be combined [8]. Pixel offsets are used to place each tissue's MS data onto a common image plane. The imaging MS datasets are then unfolded into a collated list of pixel-associated-spectra (pixel coordinates within the common image plane).

The simultaneous multivariate analysis of a tissue series containing multiple patient tissue samples enables three distinct sources of variance to be distinguished. For example, for the differentiation of two tumors:

- intratumor variability within each patient tissue

- inter-patient variability within each tumor class (endogenous variability + measurement variability)

- variation between the two tumors

An estimate of the first two sources of variance will be essential in order to establish if the observed differences between the two tumors are statistically significant.

One of the challenges facing the clinical application of imaging MS based molecular histology, whether human samples or from animal model investigations (3D imaging MS, multiple time points, multiple MS and animal repeats), is the sheer size and dimensionality of the data. An imaging MS analysis of a single tissue section typically contains $>10 \mathrm{k}$ pixels, each with an associated mass spectrum. A 50tissue patient series of $1 \mathrm{~cm}^{2}$ tissue sections, imaged with $100 \mu \mathrm{m}$ spatial resolution, would contain half a million mass spectra. High performance computer processing is necessary to efficiently process such large, multidimensional datasets.

The increased processing power provided by GeneralPurpose computing on Graphics Processing Units (GPGPU) provides a cost-effective and low-energy solution for the data analysis capabilities required for imaging MS-based molecular histology of larger tissue series or higher spatial resolution data sets. GPUs excel at tackling large amounts of similar data if the problem can be split into hundreds or thousands of pieces and calculated simultaneously. When combined with CPUs, which are adept at serial-based tasks such as data organization, the end result is a tremendous speed-up in overall system performance. The increased processing speeds provided by GPUs have recently been exploited in mass spectrometry-based proteomics to increase the speed of comparing an MS/MS spectrum with all of the potential matches from a sequence database [11], and faster $(200 \times)$ and improved feature detection using wavelet decomposition [12].

Off-the-shelf systems are available that combine high processing speeds ( $>1$ TFLOPS) and abundant on-board memory, with architectures (e.g., CUDA) that make GPUs increasingly more suitable for commercial programming and mathematical packages such as Matlab. Furthermore, it has been shown that the performance benefit of GPU processing increases with increasing matrix size (within memory limits); the large matrices generated by imaging MS of a series of tissue sections ( $>100 \mathrm{k}$ of pixels and mass spectra) are very well suited to GPU computing. Here we demonstrate the significant, $>10 \times$, improvements in processing speed that can be obtained by exploiting commercially available GPU processing boards. The greatly increased processing speeds and cost-effectiveness of GPU boards make them an excellent option for the further development of imaging MS as a novel molecular histology tool.

\section{Methods}

\section{Computer Hardware and Software}

A Dell Precision T7500 workstation equipped with a single 6core Intel Xeon X5650 2.66 GHz CPU, 48 GB DDR3 RAM (1333 MHz), and an NVidia Tesla C2070 GPU processing board, running on 64-bit windows 7, was used. Calculations were performed using 64 bit MatLab v. 7.12.0 (R2011a), and the Matlab toolboxes Bioinformatics (v. 3.7), Image Processing (v. 7.2), Parallel Computing (v. 5.1), Signal Processing (v. 6.15), and Statistics (v. 7.5). A GPU engine for Matlab, Jacket (v.1.8.0, Accelereyes), was used to convert the single threaded Matlab scripts to GPU-enabled code (www.accelereyes.com).

\section{Imaging MS Datasets}

A number of different imaging MS datasets were used to test the performance of the GPU processing board for imaging MS-based molecular histology:

(1) Mouse brain tissue series: neuropeptide imaging MS datasets of five coronal mouse brain tissue sections, imaged with $200 \mu \mathrm{m}$ spatial resolution using a Ultraflex MALDITOF/TOF (Bruker Daltonics, Bremen, Germany) and 2,5dihydroxy benzoic acid as the matrix. The project consists of 8976 pixels and a total raw data load of $2.1 \mathrm{~GB}$.

(2) Chondrosarcoma tissue series: protein imaging MS datasets of five central chondrosarcoma and five secondary peripheral chondrosarcoma, imaged with $200 \mu \mathrm{m}$ spatial resolution using a Bruker Autoflex II MALDI-TOF and $\alpha$-cyano-4-hydroxycinnamic acid as the matrix [13] (for these bone tumors it was found that a-cyano-4-hydroxycinnamic acid provided better protein mass spectra than the more common protein matrix sinapinic acid). The project consists of 28201 pixels and a total raw data load of 9.8 GB.

(3) Rat brain tissue section: neuropeptide imaging MS dataset of a coronal rat brain tissue section, imaged with $100 \mu \mathrm{m}$ spatial resolution using a Bruker UltrafleXtreme 
MALDI-TOF/TOF and $\alpha$-cyano-4-hydroxycinnamic acid as the matrix. The raw dataset consists of 19908 pixels and a total raw data load of 6.3 GB.

\section{Mass Spectral Processing and Data Reduction}

Each pixel's mass spectrum was processed using a smoothing and baseline subtraction routine using FlexAnalysis (Bruker Daltonics, Bremen, Germany). For protein imaging MS, a Gaussian algorithm was used for mass spectral smoothing (width $2 \mathrm{~m} / \mathrm{z}, 4$ cycles) and the ConvexHullV3 algorithm used for baseline subtraction. For neuropeptide imaging MS a SavitskyGolay algorithm was used for mass spectral smoothing (width $0.02 \mathrm{~m} / \mathrm{z}, 2$ cycles) and the TopHat algorithm used for baseline subtraction.

An automated mass-spectral feature detection algorithm was then applied to each imaging MS dataset [14]. For single tissue analyses the intensity of each peak was then extracted from each pixel's mass spectrum to reduce the large imaging MS datasets to much smaller image cubes that only contain the images of well-described MS peaks [14]. All GPU processing was performed on these reduced datasets.

For the analysis of projects containing imaging MS datasets of multiple tissues the mass-spectral feature detection routine was first applied to each individual dataset. The resulting dataset-specific peak lists were then collated into a final project-specific peak list (mass accuracy $\pm 500 \mathrm{ppm}$ ), which was then used to extract all features from each imaging MS dataset [8]. The reduced imaging MS datasets were then merged into a single project dataset using pixel offsets [8]. In this manner the multivariate techniques could be simultaneously applied to all imaging MS datasets in the project.

After data reduction with the automated feature detection and extraction routine the $2.1 \mathrm{~GB}$ imaging MS project of the mouse brain tissue series was reduced to a $33 \mathrm{MB}$ image cube containing the images of 476 MS features; the 9.8 GB imaging MS project of the chondrosarcoma tissue series was reduced to a $92 \mathrm{MB}$ image cube containing the images of $427 \mathrm{MS}$ features; and the 6.3 GB imaging MS dataset of the rat brain tissue section was reduced to a $49 \mathrm{MB}$ image cube containing the images of $325 \mathrm{MS}$ features. Note: data reduction via automated peak identification and extraction has the disadvantage that peaks below the signal-to-noise $(\mathrm{S} / \mathrm{N})$ threshold, but which may contribute to the differentiation, are not included in the subsequent data analysis. While a lower $\mathrm{S} / \mathrm{N}$ threshold may be used, this can lead to a rapid increase in the amount of chemical noise retained in the dataset, which can undermine the ability of the data analysis tools to differentiate between the different regions of the imaging MS datasets. The lower data loads provided by data reduction are essential to the practical application of imaging MS-based molecular histology [14].
Table 1. Data load, number of variables, and processing time as a function of series size and spatial resolution (before and after data reduction)

\begin{tabular}{|c|c|c|c|c|c|c|}
\hline & \multicolumn{2}{|c|}{ Small, low res. } & \multicolumn{2}{|c|}{ Large, low res. } & \multicolumn{2}{|c|}{ Large, high res. } \\
\hline & Raw & Proc. & Raw & Proc. & Raw & Proc. \\
\hline No. of tissues & 10 & & 100 & & 100 & \\
\hline Tissue size $\left(\mathrm{cm}^{2}\right)$ & 2 & & 2 & & 2 & \\
\hline Resolution $(\mu \mathrm{m})$ & 100 & & 100 & & 25 & \\
\hline Pixels (spectra) & $200 \mathrm{k}$ & $200 \mathrm{k}$ & $2 \mathrm{M}$ & $2 \mathrm{M}$ & $32 \mathrm{M}$ & $32 \mathrm{M}$ \\
\hline spectral channels & $70 \mathrm{k}$ & 150 & $70 \mathrm{k}$ & 150 & $70 \mathrm{k}$ & 150 \\
\hline Dataload (GB) & 52.15 & 0.22 & 521.54 & 2.24 & 8344.65 & 35.76 \\
\hline FLOPs & $1 \mathrm{E}+16$ & $6 \mathrm{E}+10$ & $1 \mathrm{E}+17$ & $6 \mathrm{E}+11$ & $2 \mathrm{E}+18$ & $1 \mathrm{E}+13$ \\
\hline Proc. time ${ }^{\#}(\mathrm{~s})$ & $201 \mathrm{k}$ & 0.9 & $2 \mathrm{M}$ & 9.0 & $31 \mathrm{M}$ & 144 \\
\hline Proc. time $(\mathrm{d})$ & 2.3 & & 22.7 & & 363 & \\
\hline
\end{tabular}

\#Using a3.8 GHz i7, 70 GFLOPS maximum processing speed.

${ }^{\mathrm{a}}$ Number of floating point operations (FLOPs) given for a common PCA algorithm, where FLOPs $=14 \cdot \mathrm{k} \cdot N^{2}+8 \cdot \mathrm{N}^{3}, \mathrm{k}$ is the number of pixels, $N$ the number of channels [18].

\section{Statistical Analysis Implementation}

The reduced data is extracted as a $2 \mathrm{D}$ matrix with the extracted peak intensities from each pixel arranged in rows and normalized to each pixel's total-ion-count. A second matrix contains the coordinates of each pixel. Principal component analysis was performed using the princomp routine from the Matlab statistics toolbox without modification. Non-Negative Matrix Factorization is based on David Ross's (University of Toronto: http://www.cs.toronto.edu/ dross/) implementation of Lee and Seung's Non-Negative Matrix Factorization algorithm [15]. For determining the correlation between images, the images were unfolded into onedimensional vectors and the Pearson correlation between the vectors calculated.

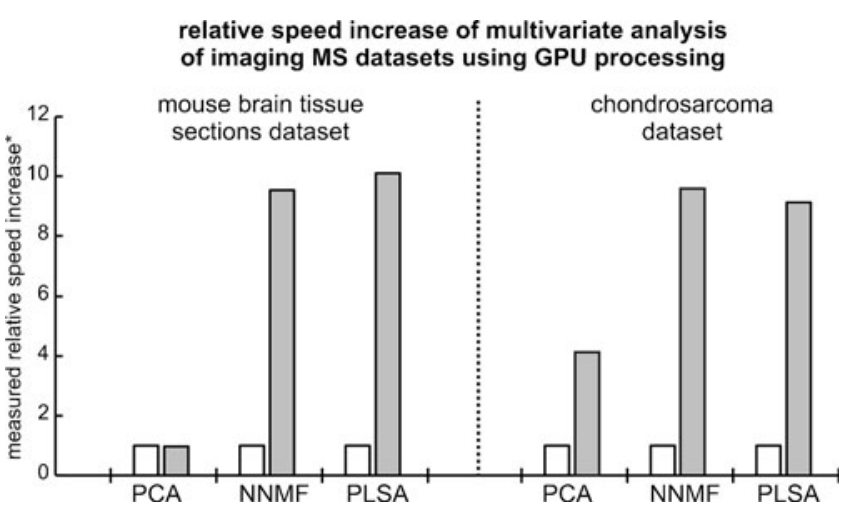

Figure 1. Relative processing speed of multivariate analysis using the Tesla C2070 GPU (grey bars) versus the Xeon X5650 CPU (white bars). The mouse brain tissue sections dataset contains the reduced imaging MS data of five coronal mouse brain tissues, and consists of 476 discrete MS peaks extracted from 8976 pixels. The larger chondrosarcoma dataset contains the reduced imaging MS data of 10 chondrosarcoma tissue sections, and consists of 427 peaks extracted from 28,201 pixels 


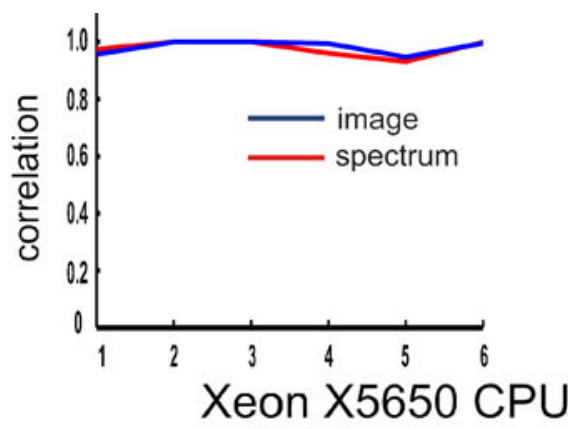
16.34 seconds
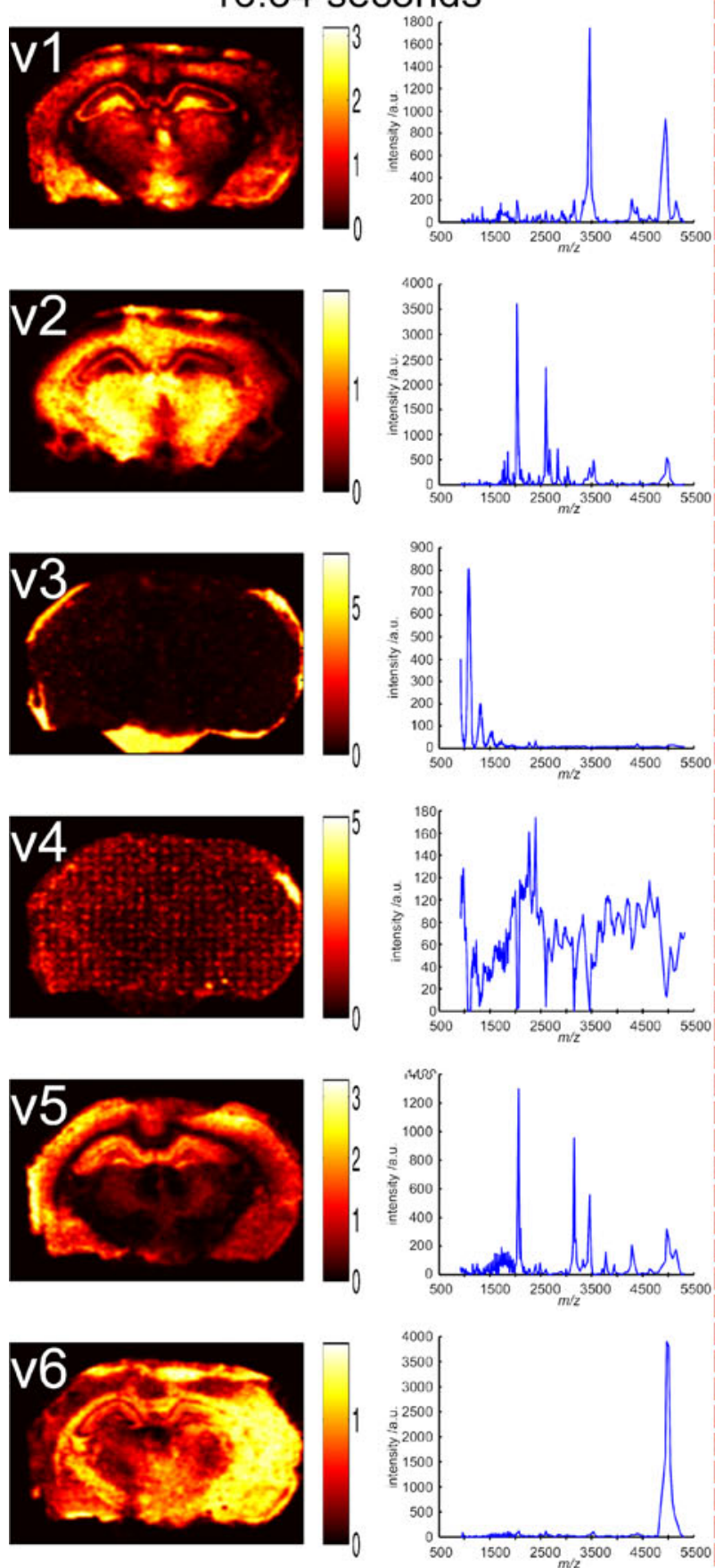

\section{Comparison of NNMF using CPU and GPU processing}

6 factors, 100 iterations

$158 \times 126$ pixel, 325 peaks; $51 \mathrm{MB}$
C2070 GPU

1.8 seconds
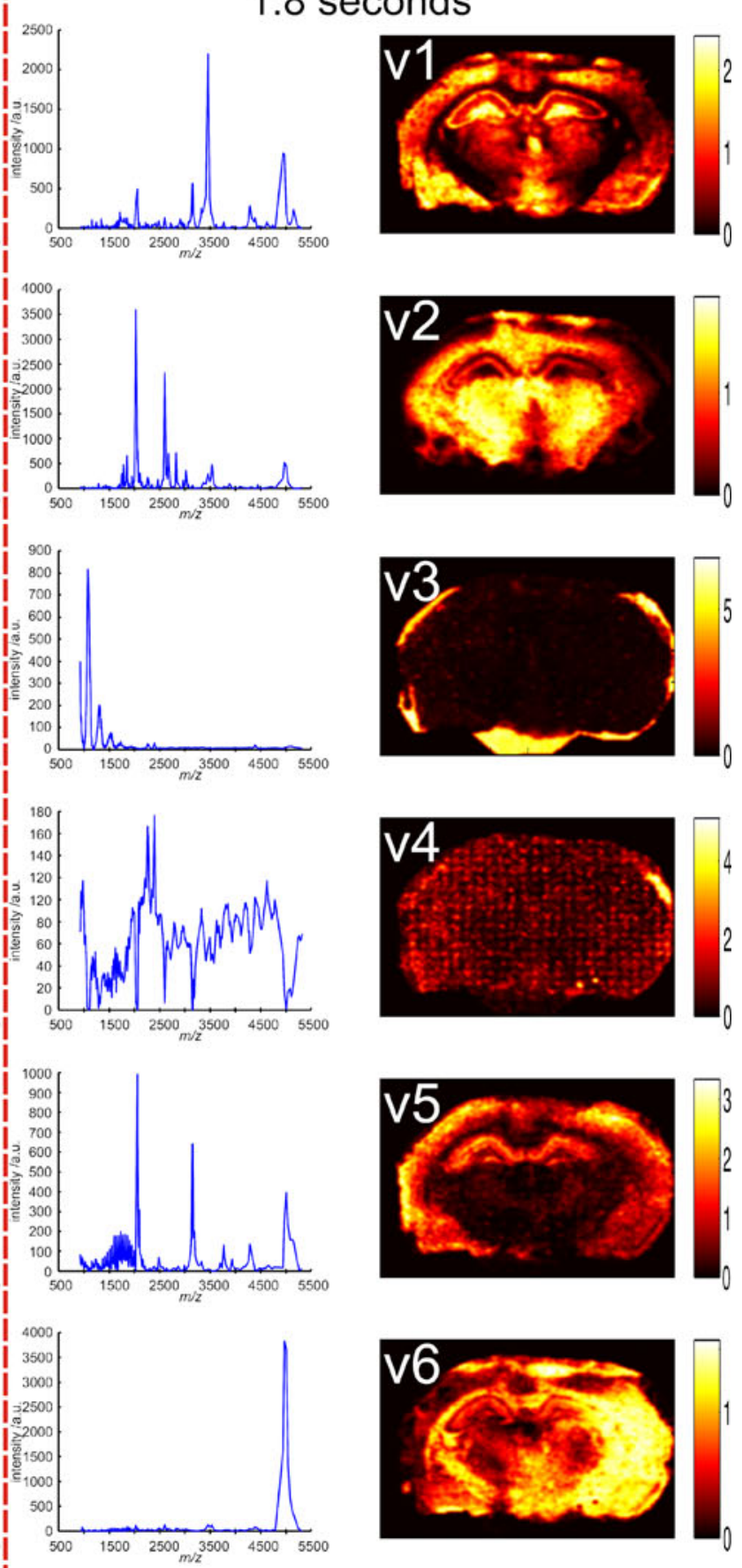

Figure 2. Comparison of images and loading plot outputs obtained by NNMF analysis of a rat brain tissue section using the Xeon X5650 CPU and the C2070 GPU. Reduced imaging MS data consisted of 325 peaks from 19,908 pixels 
Table 2. Processing times for non-negative matrix factorization of model imaging MS datasets as a function of number of pixels and number of peaks retained in the reduced imaging MS dataset. Model data consists of random double precision matrices

\begin{tabular}{lcccc}
\hline 'Pixels' $\times$ 'peaks' & Load (MB) & C2070 (s) & X5650 (s) & Improvement \\
\hline $2,000 \times 100$ & 1.6 & 0.17 & 0.49 & 2.88 \\
$10,000 \times 100$ & 8 & 0.57 & 2.44 & 4.28 \\
$50,000 \times 100$ & 40 & 2.56 & 11.80 & 4.61 \\
$100,000 \times 100$ & 80 & 10.33 & 46.33 & 4.48 \\
$500,000 \times 100$ & 400 & 25.81 & 115.61 & 4.48 \\
$1500,000 \times 100$ & 1200 & 79.16 & 362.50 & 4.58 \\
$2000,000 \times 100$ & 1600 & 321.01 & 470.92 & 1.47 \\
$500,000 \times 150$ & 600 & 29.64 & 184.83 & 6.24 \\
$500,000 \times 300$ & 1200 & 46.95 & 354.32 & 7.55 \\
$100,000 \times 150$ & 120 & 5.75 & 36.33 & 6.32 \\
$100,000 \times 300$ & 240 & 7.87 & 70.91 & 9.01 \\
$100,000 \times 500$ & 400 & 10.51 & 118.93 & 11.32 \\
$100,000 \times 800$ & 640 & 16.36 & 193.07 & 11.80 \\
$100,000 \times 1200$ & 960 & 21.71 & 284.54 & 13.11 \\
$100,000 \times 1500$ & 1200 & 26.83 & 354.76 & 13.22 \\
$100,000 \times 2000$ & 1600 & 251.40 & 462.52 & 1.84 \\
\hline
\end{tabular}

\section{Results and Discussion}

The data load and estimated data processing times, using a single high performance processor for principal component analysis (PCA) of a tissue series are provided in Table 1 as a function of series size (number of tissues) and spatial resolution, for both raw data (containing all mass spectral channels) and data that has been reduced via an automated mass-spectral feature identification and extraction routine [14]. The estimated PCA data processing times are close to that obtained in reality. It was estimated that the reduced dataset from a single intermediate grade myxofibrosarcoma tissue section would require $0.1 \mathrm{~s}$, in practice the analysis took $0.3 \mathrm{~s}$, indicating the increase in processing times with an increasing number of tissue samples or spatial resolution will be substantial. This is especially true of the data analysis techniques that have been shown to provide improved data analysis capabilities but which are computationally more demanding $[8,16]$. The computation times for the analysis of the same reduced data with k-means clustering (3.1 s), maximum autocorrelation factor analysis $(6.7 \mathrm{~s})$, nonnegative matrix factorization (18.3 s), hierarchical clustering $(41.7 \mathrm{~s})$, fuzzy c-means $(34.3 \mathrm{~s})$, and probabilistic latest semantic analysis $(27.1 \mathrm{~s})$ are all substantially longer than PCA. The application of these data analysis algorithms to tissue series containing many tissue samples or higher spatial resolution analyses (containing many more pixels) will require increased processing speeds.

Figure 1 shows the speed improvements that can be obtained for multivariate analysis of reduced imaging MS tissue series' using a single NVidia Tesla C2070 GPU processing board, which contains 448 CUDA cores and 6 GB of onboard memory. The mouse brain project contains the reduced (see the Methods section) imaging MS data from five coronal mouse brain tissue sections and consists of the intensities of 476 discrete peaks extracted from 8976 pixels and saved as double precision values, total data load 33 MB. Using the C2070 GPU board NNMF and PLSA was performed in $1.11 \mathrm{~s}$ and $5.89 \mathrm{~s}$, as opposed to $10.58 \mathrm{~s}$ and $59.50 \mathrm{~s}$, using the Xeon X5650 processor (a high performance i7 6-core processor, using all six cores), a 10-fold increase in data processing speed. The chondrosarcoma project contains reduced imaging MS datasets of five central chondrosarcoma and five secondary peripheral chondrosarcoma patient tissues, and contains more pixels and a greater data load. The reduced dataset contains the intensities of 427 discrete peaks, saved as double precision values, extracted from 28,201 pixels, total data load $92 \mathrm{MB}$. Using the C2070 GPU board NNMF and PLSA of the reduced chondrosarcoma dataset was performed in $11.52 \mathrm{~s}$ and 57.73 s, whereas with the Xeon X5650 CPU it required $109.59 \mathrm{~s}$ and $523.07 \mathrm{~s}$; again a near 10 -fold increase in processing speed.
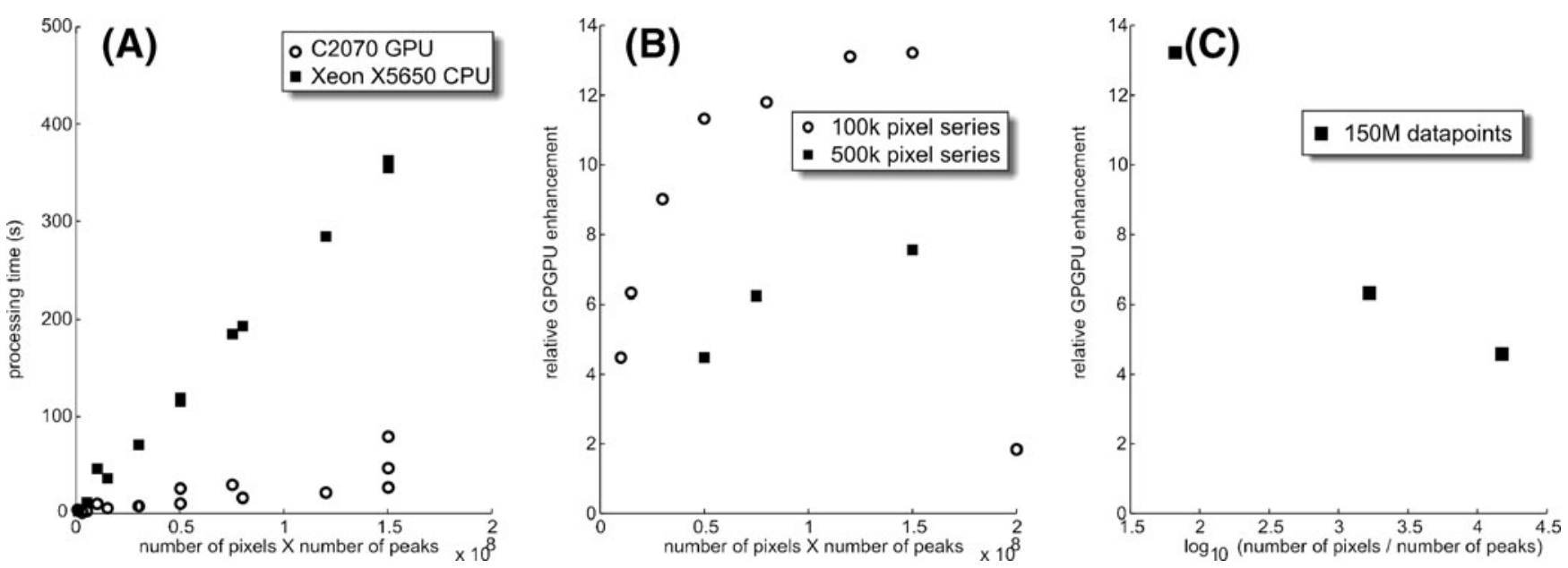

Figure 3. (A) Comparison of processing times for NNMF of model matrices using the X5650 CPU and the C2070 GPU board, B) GPU processing speed improvement for the $100 \mathrm{k}$ and $500 \mathrm{k}$ pixel datasets, and $\mathbf{C}$ ) effect of matrix rectangularity on GPU processing speed improvement for the $150 \mathrm{M}$ datapoint datasets 
PCA is a much faster data analysis algorithm, so the benefit of using GPU processing only became apparent with the larger chondrosarcoma dataset because of the time penalty of transferring the data to the GPU board. Using the GPU board PCA of the mouse brain and chondrosarcoma projects took $1.02 \mathrm{~s}$ and $4.99 \mathrm{~s}$, whereas with the Xeon X5650 CPU it required $0.99 \mathrm{~s}$ and $10.8 \mathrm{~s}$, respectively. For the smaller mouse brain dataset the time penalty required for transferring data to the GPU board caused the PCA algorithm to be faster on the Xeon X5650 processor.

The results of PCA analysis using the GPU processing board were identical to those obtained using the Xeon X5650 processor; for NNMF the results were largely identical. Figure 2 shows a comparison of the NNMF component outputs of a neuropeptide imaging MS dataset of a rat brain tissue section using the Xeon X5650 CPU and the C2070 GPU board. The Pearson correlation values indicate that the images and loading plots for each component output are very highly correlated (but sometimes not identical). Close examination of the images and loading plots reveals that any differences are small and characterized by different relative intensities. NNMF is an iterative factorization process - the small differences in the results obtained using the CPU and GPU reflect the different starting points of the factorization process.

The NVidia Tesla C2070 contains 6 GB of onboard memory. Data transfer to the GPU processing board is slow, for example $0.638 \mathrm{~s}$ for transferring the reduced chondrosarcoma data to and from the GPU. Consequently optimum performance of GPU processing is maintained only if calculations are performed with minimal data transfer between the GPU board and the main system RAM.

To ascertain the data load that could be analyzed with the GPU board while maintaining high processing speed model datasets consisting of random double-precision matrices were constructed and the NNMF processing times determined as a function of length (number of pixels in an imaging MS experimental series) and width (number of peaks) using the GPU processing board and the Xeon X5650 CPU, Table 2. GPU processing provided significantly increased processing speeds for reduced imaging MS data sets up to and including 1.2 GB. The 1.2 GB is the memory required to store the reduced imaging MS datasets; additional memory is required to perform the multivariate

Table 3. Data load and rectangularity of reduced imaging MS datasets as function of spatial resolution and number of tissue sections in tissue series. Combinations highlighted in grey can be analyzed with high speed using the Tesla C2070 GPU board

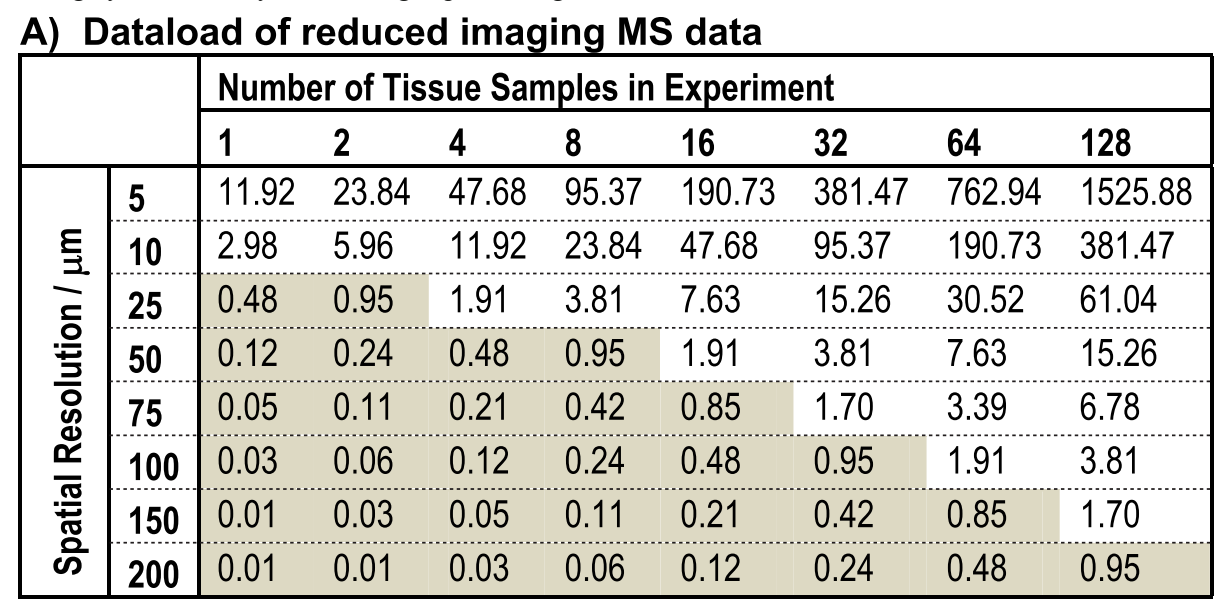

B) $\log _{10}$ (rectangularity) of reduced imaging MS data

\begin{tabular}{|c|c|c|c|c|c|c|c|c|c|}
\hline & \multicolumn{8}{|c|}{ Number of Tissue Samples in Experiment } \\
\hline & & 1 & 2 & 4 & 8 & 16 & 32 & 64 & 128 \\
\hline \multirow{8}{*}{$\begin{array}{l}\text { 营 } \\
\text { की }\end{array}$} & 5 & 4.0 & 4.3 & 4.6 & 4.9 & 5.2 & 5.5 & 5.8 & 6.1 \\
\hline & 10 & 3.4 & 3.7 & 4.0 & 4.3 & 4.6 & 4.9 & 5.2 & 5.5 \\
\hline & 25 & 2.6 & 2.9 & 3.2 & 3.5 & 3.8 & 4.1 & 4.4 & 4.7 \\
\hline & 50 & 2.0 & 2.3 & 2.6 & 2.9 & 3.2 & 3.5 & 3.8 & 4.1 \\
\hline & 75 & 1.6 & 1.9 & 2.2 & 2.6 & 2.9 & 3.2 & 3.5 & 3.8 \\
\hline & 100 & 1.4 & 1.7 & 2.0 & 2.3 & 2.6 & 2.9 & 3.2 & 3.5 \\
\hline & 150 & 1.0 & 1.3 & 1.6 & 1.9 & 2.2 & 2.6 & 2.9 & 3.2 \\
\hline & 200 & 0.8 & 1.1 & 1.4 & 1.7 & 2.0 & 2.3 & 2.6 & 2.9 \\
\hline
\end{tabular}


analysis. The memory required to perform multivariate analysis of datasets greater than $1.2 \mathrm{~GB}$ leads to data transfer between the system RAM and the GPU board, impeding the apparent processing speed.

Figure 3A shows a graphical representation of Table 2, in which the abscissa is the total number of variables (number of pixels $\times$ number of peaks). It can be seen that the processing time using the Xeon CPU and the GPU increase approximately linearly with the total number of variables, but the rate of increase for the GPU is much less. The apparent scatter in the GPU processing times is related to the rectangularity of the 2D data matrices (number of pixels/ number of peaks). Figure 3B shows the relative enhancement of GPU processing, with respect to the Xeon X5650 CPU, for the 100 and 500 pixel series of Table 2. It can be seen that the enhancement increases with an increasing total number of variables, which corresponds to an increasing number of MS peaks, until the memory limitations of the GPU card are reached. The enhancement of the $500 \mathrm{k}$ pixel series is less because of the increased rectangularity of the data matrix. Figure $3 \mathrm{c}$ presents the relative enhancement of GPU processing for datasets containing $150 \mathrm{M}$ data points $(1.5 \mathrm{M}$ pixels $\times 100$ peaks, $500 \mathrm{k}$ pixels $\times 300$ peaks, and $100 \mathrm{k}$ pixels $\times 1500$ peaks) versus the rectangularity ratio of the matrix. Larger improvements in processing speeds are obtained with matrices of lower rectangularity.

It is instructive to consider how the above observations concern imaging MS based molecular histology of tissue series, specifically how one may design the experiment to maintain its compatibility with GPU processing. Table 3 shows the data load and rectangularity of the reduced imaging MS data as a function of spatial resolution and number of tissues (assuming tissue sections of $1 \mathrm{~cm}^{2}$ and the images of 400 peaks extracted from the raw imaging MS data).

The data from the combinations of spatial resolution and number-of-tissue sections highlighted in grey can be analyzed on the C2070 GPU board without data exchange with system RAM and have rectangularities less than 1000, thus maintaining high speed data analysis capabilities. Using $100 \mu \mathrm{m}$ spatial resolution up to forty $1 \mathrm{~cm}^{2}$ tissues can be analyzed (1.19 GB dataload). Such a scenario is expected from neuropeptide and protein imaging MS analyses (e.g., the mouse brain and chondrosarcoma imaging MS datasets used to construct Figure 1).

The detection of an increased number of distinct molecular ions, as may occur with a high mass resolution analysis of the proteolytic peptides produced via on-tissue digestion, decreases the rectangularity of the matrices but increases the data load. If 1000 distinct mass spectral features are retained in the reduced imaging MS datasets, only sixteen $100 \mu \mathrm{m}$ spatial resolution imaging MS datasets of $1 \mathrm{~cm}^{2}$ tissues could be analyzed using the C2070 GPU board (before data exchange with the system RAM).

Table 3 also indicates one of the general challenges facing high spatial resolution imaging MS analysis-the large number of pixels lead to very large data loads and highly rectangular data matrices. The data load from just four $1 \mathrm{~cm}^{2}$ tissues, analyzed with $10 \mu \mathrm{m}$ spatial resolution and including the images of 400 ions, is 11.92 GB. Previous multivariate analysis of high spatial resolution imaging MS data has used mass spectral and spatial binning, as well as limiting the analysis to specific mass ranges, to reduce the data load and to focus the analysis on the more relevant molecular information [17]. In order to maintain high spatial resolution during data analysis it will be important to parse the data as much as possible, by focusing the analysis on specific regions of interest and MS features.

The highly encouraging performance metrics reported here for imaging MS based molecular histology using GPU processing were obtained using commercial systems, and so will be underestimates of the speed improvements that can be attained with codes written explicitly for such processing. For example, Hussong et al. [12] have reported 200× speed improvements for mass spectral denoising and feature detection using wavelet based methods. Optimized programming, perennial advances in computational technology (hardware and software) and the possibility to develop cost-effective GPU clusters for super-computer processing capacity, at a fraction of the financial and environmental cost of a CPU based system (e.g. www.littlegreenmachine.org), means that there is widespread scope for further improving the data processing capacity of imaging MS datasets using GPU based systems.

\section{Conclusion}

There is increasing evidence that imaging MS based molecular histology can complement established histologic and histochemical methods by distinguishing between pathologic entities with overlapping/identical pathologies, revealing intratumor heterogeneity, and identifying tumor interface zones. The application of imaging MS based molecular histology to series of tissues, whether clinical cohorts or from animal models of disease, requires increased processing power to simultaneously analyze the large number of tissues. The high dimensionality of imaging MS data is particularly well suited to GPU processing; here it is demonstrated that improvements in processing speed up to $13 \times$ can be obtained using relatively inexpensive, commercially available systems. GPU processing represents the most cost-effective method for obtaining the high processing capacity required to apply the multivariate data analysis techniques at the heart of imaging MS-based molecular histology to the larger tissue series necessary for its clinical application and cross-validation [8].

\section{Acknowledgements}

The authors gratefully acknowledge the help of Associate Professor Judith V. M. G. Bovée for the chondrosarcoma tissues and Professor Simon Luckman for the rat brain tissue sample. This work is financially supported by NWO 
Horizon project 93511027, the ICT consortium COMMIT project e-biobanking with imaging and the Swedish Research Council, grant no. 2008-5597, 2009-6050, 20105421, 2010-0881, and the K\&A Wallenberg Foundation.

\section{References}

1. Chaurand, P., Sanders, M.E., Jensen, R.A., Caprioli, R.M.: Proteomics in diagnostic pathology-profiling and imaging proteins directly in tissue sections. Am. J. Pathol. 165, 1057-1068 (2004)

2. McDonnell, L.A., Heeren, R.M.A.: Imaging mass spectrometry. Mass Spectrom. Rev. 26, 606-643 (2007)

3. McDonnell, L.A., Willems, S.M., Corthals, G.L., van Remoortere, A., van Zeijl, R.J.M., Deelder, A.M.: Imaging mass spectrometry in cancer research: past experiences and future possibilities. J. Proteom. 73, 1921-1944 (2010)

4. Cazares, L.H., Troyer, D., Mendrinos, S., Lance, R.A., Nyalwidhe, J.O., Beydoun, H.A., Clements, M.A., Drake, R.R., Semmes, O.J.: Imaging mass spectrometry of a specific fragment of mitogen-activated protein kinase/extracellular signal-regulated kinase kinase kinase 2 discriminates cancer from uninvolved prostate tissue. Clin. Cancer Res. 15, 5541-5551 (2009)

5. Hardesty, W.M., Kelley, M.C., Mi, D., Low, R.L., Caprioli, R.M.: Protein signatures for survival and recurrence in metastatic melanoma. J. Proteom. 74, 1002-1014 (2011)

6. Willems, S.M., van Remoortere, A., van Zeijl, R., Deelder, A.M., McDonnell, L.A., Hogendoorn, P.C.W.: Imaging mass spectrometry of myxoid sarcomas identifies proteins and lipids specific to tumor type and grade, and reveals biochemical intratumor heterogeneity. J. Pathol. 222, 400-409 (2010)

7. Deininger, S.-O., Ebert, M.P., Fütterer, A., Gerhard, M., Röcken, C. MALDI imaging combined with hierarchical clustering as a new tool for the interpretation of complex human cancers. J. Proteome Res. 7, 5230-5236 (2008)

8. Jones, E.A., van Remoortere, A., van Zeijl, R.J.M., Hogendoorn, P.C. W., Boveé, J.V.M.G., Deelder, A.M., McDonnell, L.A.: Multiple statistical analysis techniques corroborate intratumor heterogeneity in imaging mass spectrometry datasets of Myxofibrosarcoma. PLoS ONE 6, e24913 (2011)

9. Kang, S., Shim, H.S., Lee, J.S., Kim, D.S., Kim, H.Y., Hong, S.H., Kim, P.S., Yoon, J.H., Cho, N.H.: Molecular proteomics imaging of tumor interfaces by mass spectrometry. J. Proteome Res. 9, 1157-1164 (2010)

10. Oppenheimer, S.R., Mi, D., Sanders, M.E., Caprioli, R.M.: Molecular analysis of tumor margins by MALDI mass spectrometry in renal carcinoma. J. Proteome Res. 9, 2182-2190 (2010)

11. Baumgardner, L.A., Shanmugam, A.K., Lam, H., Eng, J.K., Martin, D. B.: Fast parallel tandem mass spectral library searching using GPU hardware acceleration. J. Proteome Res. 10, 2882-2888 (2011)

12. Hussong, R., Gregorius, B., Tholey, A., Hildebrandt, A.: Highly accelerated feature detection in proteomics data sets using modern graphics processing units. Bioinformatics 25, 1937-1943 (2009)

13. Jones, E.A., Waaijer, C., Schmitz, N., Freser, C., Remoortere, A.v., Zeijl, R.J.M.v., Hogendoorn, P.C.W., Heck, A.J.R., Deelder, A.M., Altelaar, A.F.M., Bovée, J.V.M.G., McDonnell, L.A. Differentiation of Morphologically Identical Tumors Using Imaging Mass Spectrometry Based Molecular Histology, unpublished (in preparation 2011)

14. McDonnell, L.A., van Remoortere, A., de Velde, N., van Zeijl, R.J.M., Deelder, A.: Imaging mass spectrometry data reduction: automated feature identification and extraction. J. Am. Soc. Mass Spectrom. 21, 1969-1978 (2010)

15. Lee, D.D., Seung, H.S.: Learning the parts of objects by non-negative matrix factorization. Nature 401, 788-791 (1999)

16. Hanselmann, M., Kirchner, M., Renard, B.Y., Amstalden, E.R., Glunde, K., Heeren, R.M.A., Hamprecht, F.A.: Concise representation of mass spectrometry images by Probabilistic Latent Semantic Analysis. Anal. Chem. 80, 9649-9658 (2008)

17. Broersen, A., van Liere, R., Altelaar, A.F.M., Heeren, R.M.A., McDonnell, L.A.: Automated, feature-based image alignment for high-resolution imaging mass spectrometry of large biological samples. J. Am. Soc. Mass Spectrom. 19, 823-832 (2008)

18. Golub, G.H., van Loan, C.F.: Matrix Computations. John Hopkins University Press, Baltimore (1996) 